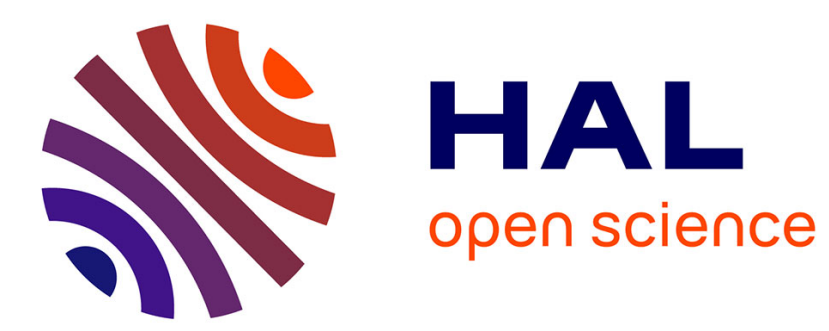

\title{
NEW ASPECTS OF THE FLUID LOADING OF VIBRATING STRUCTURES
}

\author{
D. Crighton
}

\section{To cite this version:}

D. Crighton. NEW ASPECTS OF THE FLUID LOADING OF VIBRATING STRUCTURES. Journal de Physique IV Proceedings, 1992, 02 (C1), pp.C1-581-C1-589. 10.1051/jp4:19921126 . jpa00251082

\section{HAL Id: jpa-00251082 https://hal.science/jpa-00251082}

Submitted on 1 Jan 1992

HAL is a multi-disciplinary open access archive for the deposit and dissemination of scientific research documents, whether they are published or not. The documents may come from teaching and research institutions in France or abroad, or from public or private research centers.
L'archive ouverte pluridisciplinaire HAL, est destinée au dépôt et à la diffusion de documents scientifiques de niveau recherche, publiés ou non, émanant des établissements d'enseignement et de recherche français ou étrangers, des laboratoires publics ou privés. 


\title{
NEW ASPECTS OF THE FLUID LOADING OF VIBRATING STRUCTURES
}

\author{
D.G. CRIGHTON \\ Head of the Deparment of Applied Mathematics \& Theoretical Physics, University of Cambridge, \\ Silver Street, GB-Cambridge CB3 9EW, Great-Britain
}

This paper will review recent work on two particular aspects of the response of fluid-loaded elastic structures. The first concerns the response of a simple bending plate to concentrated mechanical excitation with inclusion of the effects of uniform fluid flow past the plate. Many new results are found, the principal implication of them being that mean fluid flow leads to a completely different energy flow between the excitation, the mean flow and the various wave modes in the system. The second concerns the response of a mechanically-driven structure subject to fluid loading by static fluid, supported by a large number of stiffeners, or ribs, wilch may have periodic or random arrangement. Both isolated and extensive random:'ss are considered, and among the effects demonstrated are t'iose associated with expurential Anderson localization, and its short-circuiting by long-range fluid coupling.

Cet article donne un compte rendu de travaux recents sur deux aspects particuliers de la réaction des structures élastiques soumisent à une charge fluide. Le premier concerne la réaction d'une simple tôle pliante à une excitation mécanique concentrée tout en considérant l'inclusion des effets d'un écoulement fluide uniforme sur la tôle. Un grand nombre de nouveau résultats a été établi, l'implication principale étant que l'écoulement moyen d'un fluide conduit à un écoulement d'énergie complètement different entre l'excitation, l'écoulement moyen et les divers modes d'ondes présents dans le système. Le deuxième concerne la réaction d'une structure actionée mécaniquement, qui est soumise à une charge de fluide statique et supportée par un grand nombre de durcisseurs, ou baleines, disposés d'une manière périodique ou aléatoire. Une disposition aléatoire locale et étendue sont tous les deux étudićes, et parmi les effets dérivés sont ceux qui sont associés à la localisation exponentielle d'Anderson et son court-circuitage par l'accouplement fluide $\grave{c}$. longue portée. 


\section{Fluid loading with mean flow}

Here we briefly summarise results recently obtained (Refs. 1,2) for the response of simple fluid-loaded structures to localised mechanical excitation with inclusion of the effects of uniform mean fluid flow over the structure. The structure is taken as a thin elastic plate, and the fluid as strictly incompressible and inviscid. Excitation is provided by a line force, imposed along the $z$-axis and acting in the $y$-direction on the plate which occupies the $(x, z)$ plane. The fluid occupies the half-space $y>0$, with a vacuum in $y<0$, and the fluid has uniform mean velocity $U$ over the plate, in the $x$-direction. We are interested in single-frequency forcing, with the line force given by $F(t)=F_{0} \exp \left(-i \omega_{0} t\right)$, but because the system may sustain instabilities it is necessary to examine causality issues carefully, and to seek the long-time limit of a causal start-up problem. with $F(t)=F_{0} \exp \left(-i \omega_{0} t\right) H\left(t-t_{0}\right)$.

If $\phi(x, y, t)$ is the potential for the unsteady fluid motion, $p(x, y, t)$ the pressure, $m, B$ the plate specific mass and bending stiffness, $\rho_{0}$ the fluid density, then for an infinite plate the boundary value problem to be solved is

$$
\begin{gathered}
\Delta \phi=0(y>0) ; \frac{\partial \phi}{\partial y}(x, 0, t)=\frac{\partial \eta}{\partial t}+U \frac{\partial \eta}{\partial x} \\
p=-\rho_{0}\left(\frac{\partial \phi}{\partial t}+U \frac{\partial \phi}{\partial x}\right) ; m \frac{\partial^{4} \eta}{\partial t^{2}}+B \frac{\partial^{4} y^{\prime}}{\partial x^{4}}=F(t) \delta(x)-p(x, 0, t),
\end{gathered}
$$

with decay conditions as $y \rightarrow+\infty, \eta(x, t)$ being the plane deflexion in the $y$-direction. This system can be made dimensionless in such way that $\rho_{0}, m, B$ are all replaced by unity and only a single dimensionless velocity $U$ remains.

A formal causal solution (i.e. $\eta \equiv 0$ for $t<t_{0}$ if $F(t) \equiv 0$ for $t<t_{0}$ ) can be obtained by Fourier transforms as

$$
\begin{aligned}
\eta(x, t) & =\frac{1}{4 \pi^{2}} \int_{\Gamma} \Psi(x, \omega) \bar{F}(\omega) e^{-i \omega t} d \omega, \\
\Psi(x, \omega) & =\int_{C} \frac{e^{i k x}}{D(k, \omega)} d k \\
D(k, \omega) & =\left(k^{4}-\omega^{2}\right)-(\omega-U k)^{2} /|k| .
\end{aligned}
$$

Here $\vec{F}(\omega)$ is the transform of $F(t)$, the dispersion function $D(k, \omega)$ is written for real $k$, and the path of integration $C$ for the space response is the real $k$ axis. However, the path $\Gamma$ must be taken high up in the upper half $\omega$-plane: $\operatorname{Im} \omega=\omega_{i}>0$ and sufficiently large that no singularities of $\Psi(x, \omega)$ or $\bar{F}(\omega)$ lie above it. Now we attempt to reduce $\omega_{i}$ to zero, while maintaining analyticity of $\Psi(x, \omega)$. If we car. do this, then we will have a causal single-frequency response

$$
\eta(x, t)=\frac{F_{0}}{2 \pi} \Psi(x, \omega) 2^{-i \omega t}
$$

to single-frequency forcing $F(t)=F_{0} e^{-i \omega t}$ started at some definite time in the distant past. (We suppress the suffix on $\omega_{0}$ now.) But as we change $\omega_{i}$, the zeros of $D(k, \omega)$ move in the $k$-plane, and if they cross the real axis, (1.3) would jump discontinuously. Therefore $C$ must be deformed so that singularities (i.e. zeros of $D$ ) remain always on the same side of it as they originally were for $\omega_{i} \rightarrow+\infty$. Since (with $|k|$ interpreted as $k$ for $\operatorname{Re} k>0$, $-k$ for $\operatorname{Re} k<0)(1.4)$ is a quintic polynomial in each of the half-planes $\operatorname{Re} k \gtrless 0$, it is possible to follow the motion of the zeros of $\Delta$ as $\omega$ changes by a combination of analysis and simple computation. 
It is found that $\omega_{i}$ can be continuously reduced to 0 whenever

$$
\begin{aligned}
U<U_{C} & =\left(\frac{2^{\frac{1}{2}} 5^{\frac{5}{4}}}{3^{\frac{3}{4}}}\right)\left[2-\frac{1}{2}(15)^{\frac{1}{2}}\right]^{3 / 2} \\
& =0.074 .
\end{aligned}
$$

For $U<U_{C}$ there is a causal high-frequency response. For $U>U_{C}$ there is a branch point in the $\omega$-plane, at $\omega=\omega_{b}$, $\operatorname{Im} \omega_{b}>0$, with a cut running downward from $\omega_{b}$. The $\omega$-path $\Gamma$ cannot be brought down to the real axis alone; there is in addition a loop around the edges of the cut, and as $t \rightarrow+\infty$ the response in $\eta(x, t)$ is dominated by the branch point,

$$
\eta(x, t) \propto \bar{F}\left(\omega_{b}\right) t^{-1 / 2} \exp \left(i k_{b} x-i \omega_{b} t\right),
$$

where $k_{b}$ is the double wavenumber corresponding to $\omega=\omega_{b}$. Equation (1.7) represents exponential growth everywhere in time, and for $U>U_{C}$ the system is absolutely unstable, with no single-frequency behaviour as the long-time response.

Confine attention to $U<U_{C}$. Then the response is given by (1.3) and (1.5), with $C$ now deformed below any pole which has crossed the real axis from above, and above any which has crossed from below. The frequency range is now found to divide into three (with wavenumber diagrams as in fig. 1), as follows:

(1) $0<\omega<\omega_{s}(U)$ - convectively unstable range,

(2) $\omega_{s}(U)<\omega<\omega_{p}(U)$ - anomalous neutral range,

(3) $\omega_{p}(U)<\omega<\infty$ - conventional neutral range.

In each range there are four wavenumber poles, of vrhich $k_{1}^{+}, k_{2}^{+}$give rise to waves found downstream of the excitation, $k_{3}^{+}, k_{3}^{-}$to waves fourd upstream. The frequencies $\omega_{9}(U)$, $\omega_{p}(U)$ can be found numerically, or analytically for $J \ll 1$ as

$$
\begin{aligned}
\omega_{s}(U) & =U^{2}\left(1-2 U+O\left(U^{2}\right)\right) \\
\omega_{p}(U) & =N U^{5 / 3}\left(1+\frac{5}{4} N U^{2 / 3}+O\left(U^{4 / 3}\right),\right. \\
N & =\left(2^{2} 3^{3} / 5^{5}\right)^{1 / 3}
\end{aligned}
$$

In range (1) there are convectively amplifying and decaying instability waves downstream,

$$
\eta(x, t)=\frac{F_{0}}{2 U^{3 / 2} \omega^{1 / 2}} \exp \left[\frac{i \omega}{U}(x-U t)\right]\left\{\exp \left(\frac{\omega}{U}\right)^{3 / 2} x-\exp -\left(\frac{\omega}{U}\right)^{3 / 2} x\right\}
$$

and a standing wave upstream

$$
\eta(x, t)=\frac{2 F_{0}}{3 U^{2}} \sin \left(U^{2 / 3} x\right) \exp (-i \omega t)
$$


(for $U \ll 1$ ). In range (2) there are two neutral waves found downstream $\left(k_{1}^{+}, k_{2}^{+}\right)$, and two neutral waves upstream $\left(k_{3}^{+}, k_{3}^{-}\right)$. The range is anomalous because the wave $k_{2}^{+}$has negative group velocity, directed inward toward the excitation. This appears to contradict the normal outward group velocity radiation condition, out it is demanded here by causality. We argue (Ref. 1) that the usual physical basis for the group velocity condition is irrelevant here; the excitation is not the source of all energy, bur is simply a device which can transfer energy between the excitation, the various wave moles, and the mean flow, and in both ranges (1) and (2) energy is being converted from steady mean flow energy to wave energy.

In range (3) the behaviour is like that of a plate in vacuum. There is one neutral wave downstream, one evanescent wave downstrean, and a similar pair upstream, with properties qualitatively identical to those of bendiag waves on a plate in vacuum. In addition to all of these waves corresponding to pole singularities there is a field from the branch line integral in $k$-space. This decays algebraically (like $x^{-2}$ ), has no phase structure, and is significant only in the near field.
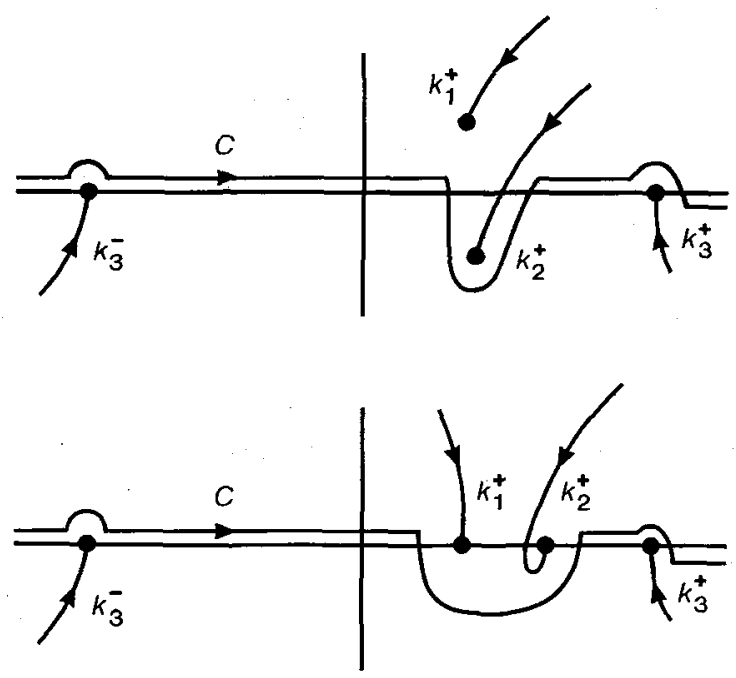

(2)
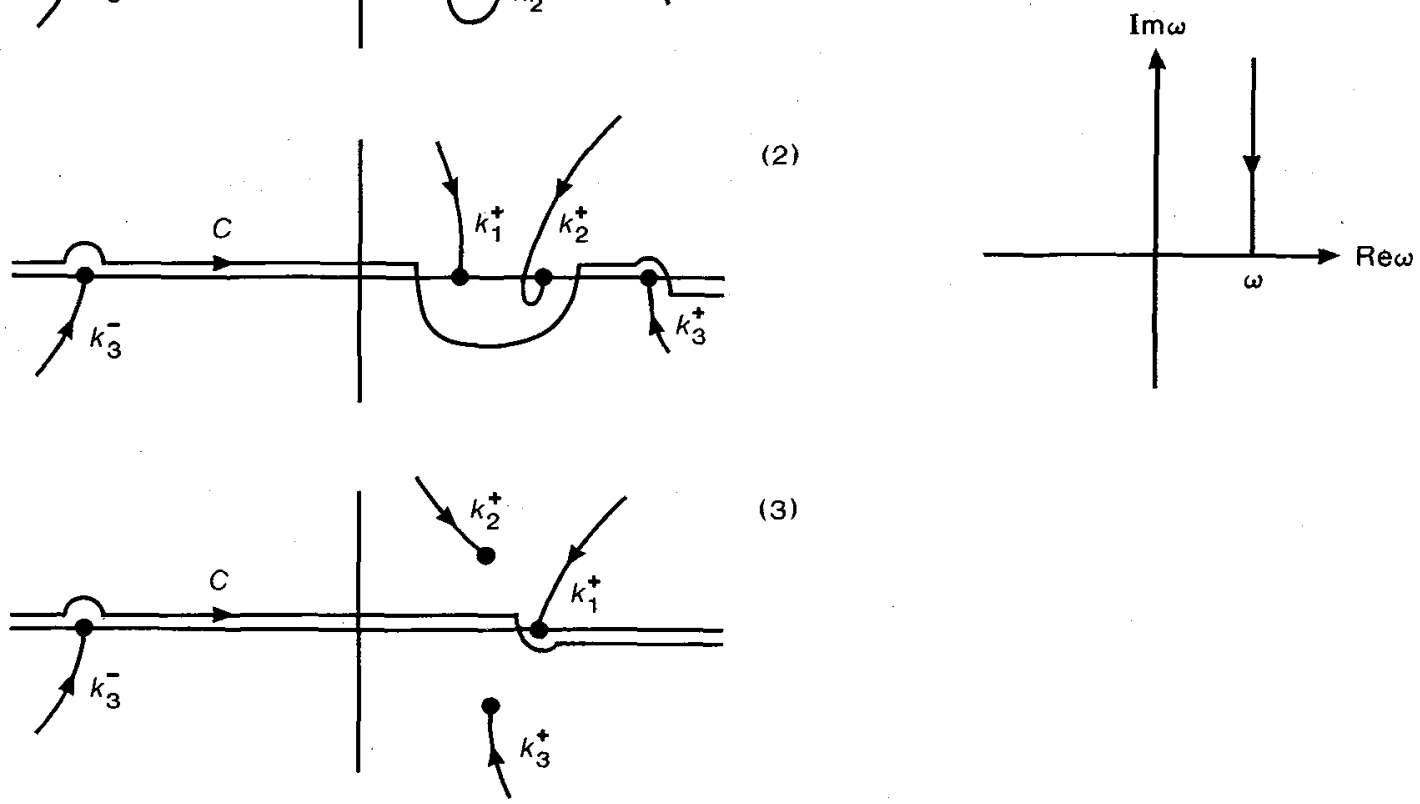

(3)

Figure 1. Passage of zeros of $D(k, \omega)$ as $\operatorname{Im} \omega$ is reduced to zero from large positive values. Numbers (1), (2), (3) refer to frequency ranges identified in the text. 
Examination of the field near the drive point also gives surprising results. We find (Ref. 1) that not only over the convectively unstable range (1) but also throughout the anomalous neutral range (2) the flow of energy is from the plate-fluid system into the excitation mechanism; if $A_{0}=-i \omega \eta(0) / F_{0}$ is the line drive ad.nittance, then we find $\operatorname{Re} A_{0}<0$ for $0<\omega \leq \omega_{s}(U)$ and $\omega_{s} \leq \omega<\omega_{p}(U)$, and the excitation mechanism providing a constant force magnitude $F_{0}$ must be prepared to absorb energy at the mean rate $(1 / 2)\left|F_{0}\right|^{2}\left|\operatorname{Re} A_{0}\right|$. The transition from range (1) to range (2) is smooth; but $\operatorname{Re} A_{0} \rightarrow-\infty$ as $\omega$ approaches $\omega_{p}$ from below, and $\operatorname{Re} A_{0}$ approaches a positive finite ron-zero limit as $\omega \rightarrow \omega_{p}$ from above. For $\omega_{p}(U) \leq \omega<\infty, \operatorname{Re} A_{0}>0$ and the power flow $\mathrm{s}$ in the conventional sense, from the excitation mechanism to the wave system.

This prompts further study of the energy balan ie in the system. For $U<U_{C}$, where there is single-frequency response, one can derive, by direct manipulation of the set (1.1), an energy equation, whose time-averaged form is

$$
J\left(X_{2}\right)=J\left(X_{1}\right)+I_{0}
$$

where $J(X)$ can be interpreted as the flux of energy, $\mathrm{n}$ the direction of increasing $x$, across station $x=X$ (in the plate and in the fluid), and $I_{6}$ is the rate of working of the external force if $x=0$ lies between $X_{1}$ and $X_{2}$. The total flus $J(X)$ is the sum of three terms,

$$
J(X)=J_{p}(X)+J_{f}(X)+J_{p f}(X),
$$

of which

$$
J_{p}(X)=\left\langle\eta_{x x x} \eta_{t}-\eta_{x x} \eta_{x t}\right\rangle
$$

and

$$
J_{f}(X)=\left\langle\int_{0}^{\infty}\left(p+U \phi_{x}\right) \phi_{x} d y\right\rangle
$$

are the familiar mechanical flux of energy in the pla:e, and the flux parallel to the surface associated with wave motion in the fluid, while

$$
J_{p f}(X)=\left\langle U \eta \phi_{t}(X, 0, t)\right\rangle
$$

is a new plate-fluid coupling flux.

With these expressions and the expressions for the plate and fluid response we have checked the way in which (1.11) is satisfied. The folowing points are important:-

(a) the flux $J(X)$ associated with a single conrectively amplifying or decaying wave is proved to be identically zero (as it must, stherwise (1.11) could not be satisfied in the convectively unstable range where amplitudes vary exponentially with $x$ );

(b) there is a fixed ( $x$-independent) flux arising from the cross-product of terms corresponding to an amplifying instability a:ld its conjugate decaying instability wave - and this cross-product is responsil: le for the entire downstream flux for $0<\omega<\omega_{s}(U)$

(c) the cross-product of an amplifying or decaying mode with any neutral mode gives zero flux:

(d) for any neutral mode, with wavenumber $\kappa$ say, the flux is given by $J=c_{g} T$, where $c_{g}$ is the group velocity for mode $\kappa$ and $T$ is the mean energy density in that mode;

(e) the various fluxes may be positive or negative, and there may be a net flux of energy towards the excitation either from l.pstream or downstream. 
Additionally we show that some of the neutri. waves in this system are negative energy waves (i.e. the energy of the system with the wave present is less than that in the undisturbed state). In particular, the anomalous neutral wave with wavenumber $k_{2}^{+}(\omega)$, which has negative group velocity even though it is found downstream of the excitation, has negative energy. Therefore its flux is positive, outward from the excitation. On the other hand, the wave $k_{1}^{+}(\omega)$, which has positive group velocity, also has negative energy, and therefore its flux is negative, inward towards the excitation, and contributes to the negative value of $\operatorname{Re} A_{0}$. Of the two waves found upstream, both have negative (i.e. outward) group velocity, but one has negative energy, the other positive. That with negative energy also carries energy inward towards the excitation.

In Ref. 2 we extend these studies to semi-infinite and finite plates (with the same uniform flow on both sides). If the plate is freely suspended, we allow for a vortex wake downstream with a strength fixed by a trailing edge Kutta condition. It is found that a wave reaching the leading edge (of a freely suspended pla;e) is reflected with greatly increased amplitude. Again, the plate edge acts as a time-dependent inhomogeneity, capable of converting mean flow energy into wave energy (or vice-versa). At a trailing edge a wave is reflected with much reduced energy - much of the energy propagating on downstream on the vortex wake. However, the leading edge gain and trailing edge loss balance each other in global terms. Then if in addition a phase condition is satisfied, a reverberant field is set up corresponding to resonant oscillations. Usually such resonance is established only in systems with no losses, waves being reflected with no more than a phase change. Here there is almost complete loss to the wake at a trailing edge, but a compensating large gain from the mean flow at a leading edge.

The lecture presented will end this section wivh a discussion of various extensions which now need to be made to this work on fluid loading with mean flow.

\section{Fluid loading of irregularly-ribbed structires}

In this section we discuss work on the forced risponse of a structure (taken to be a membrane, for simplicity), which is subject to fluid $k$ ading by static fluid, but is supported by an array of stiffeners, or "ribs". To emphasise the role of fluid loading the ribs are taken to have infinite mechanical impedance, so that fluid coupling is the only mechanism for energy transfer from some site of mechanical excitaticn from bay to bay down the structure. For definiteness it will be assumed that the rib at $x=0$ is driven with a prescribed velocity $V_{0} \exp (-i \omega t)$; all the other ribs, at $x=\zeta_{n}$ say, are fixed, with zero velocity.

Let $G(x)$ (with $\exp (-i \omega t)$ now understood wher əver appropriate) be the Green's function for the infinite homogeneous fluid-loaded structures (i.e. velocity at $x$ due to unit force at $x=0$ ). Then the velocity at any point on the ribbed structure is

$$
V(x)=\sum_{n} F_{n} G\left(x-\zeta_{n}\right)
$$

with $F_{n}$ the force exerted on the structure by the rib at $x=\zeta_{n}$. These forces are to be determined from the systern

$$
\sum_{n} F_{n} G\left(\zeta_{m}-\zeta_{n}\right)=V_{0} \delta_{m, 0}
$$


and then the response at any $x$ follows from (2.1). There are two components of $G(x)$ which lead to very different response (which may be charact, srised by the $F_{n}$ ). First $G(x)$ contains a subsonic-plane-wave component, $A_{\infty} \exp (i \kappa|x|)$ where the line transfer admittance $A_{\infty}$ and the free wavenumber $\kappa$ incorporate fluid loading. Second, $G(x)$ contains a long-range acoustic or hydrodynamic component, $B|x|^{-\nu} \exp \left(i k_{0}|x|\right)$, where the decay index $\nu$ varies from one parameter range to another, and $k_{0}=\omega / c_{0}$ is the acoustic wavenumber. In the light fluid loading régime $\nu=1 / 2$ or $3 / 2$, while in the heavy fluid loading régime $\nu=2$ and $k_{0}$ may essentially be set equal to zero.

In Ref. 3 the case of an infinite periodic rib array was considered, $\zeta_{n}=n h$. Then a formal exact solution can be obtained for the $F_{n}$ If only the subsonic wave coupling is included in $G(x)$ (this is equivalent to a nearest-neighbour-only mechanical coupling between bays), the response alternates between stop bands, $F_{n} \sim \exp (-\gamma|n|), \gamma$ real and positive, and pass bands $F_{n} \sim \exp (i \alpha|n|), \alpha$ real and positive, as the frequency varies. There is no energy input to the system in the steady state in the stop bands, but an input in the pass bands which is communicated without attenuation to infinity - the classical pattern of response in a periodic lattice. If the long-range component of $G(x)$ is also included (even though it is generally much sma!ler than the plane-wave component), the exponential decay in the stop bands is short-cirruited and replaced by slow algebraic decay. Specifically, inclusion of $B|x|^{-2}$ leads to $F_{n} \sim|n|^{-2}$, while inclusion of either $B|x|^{-1 / 2} \exp \left(i k_{0}|x|\right)$ or $B|x|^{-3 / 2} \exp \left(i k_{0}|x|\right)$ leads to $F_{n} \sim|n|^{-3 / 2} \exp \left(i k_{0} h|n|\right)$ for large $n$. These (full, detailed) analytical results have been verified in numerical study of the system (2.2) in the stop bands (see Ref. 4).

In the pass bands, however, there is completely different behaviour between the unattenuated fixed amplitude response of the strictly infinite system and what is observed in a finite system no matter how large (Ref. 4). This arises from reflections at the ends of the finite system, no matter how far away, and the waves propagating in both directions in the system interfere with each other in an extremely complicated way. Spivack (Ref. 5) has recently examined this problem, and found exact solutions for the response of a finite periodic array when subsonic plane-wave coupling is incorporated. The response in the pass bands is never that of the infinite array. Only at the mid-pass-band frequencies is the response constant in amplitude along the array, but it differs, depending on whether (with the central rib driven) the number of ribs in half of the array is even or odd, and in neither case is it equal to the infinite-array value. Away from the mid-pass-band frequencies the response is very complex, with large and rapid variation from one rib to the next, and with great sensitivity to the precise geometrical, structure and frequency parameters.

Next, the case of a single "impurity" has been examined by Sobnack (Ref. 4) using Spivack's propagation matrix method. An exact solution was found when one rib was randomly displaced from its nominal regular location. The principal result of physical interest is that there is in almost all cases a substantial reduction in the energy transmitted to bays on the other side (from the excitation) of the displaced rib. If two ribs are displaced, then numerical studies show a further reduction in the energy in bays beyond the displaced ribs. 
Sobnack and the present author (see Refs. 4,6) have also examined the case of a generally irregular rib array, with all ribs randomly displaced from their regular locations. If subsonic-wave coupling only is included (i.e. nearest-neighbour coupling of the bays) then one finds the phenomenon of Anderson localisation (see Ref. 7) familiar in solidstate physics, in which the pass-band behaviour of the regular structure is replaced by exponential localization of energy around the source of excitation. The localization length has been calculated (Ref. 4) in both the small and large randomness limits, with good agreement with the results of numerical experiments in which inversions of the set (2.2) were carried out for thousands of realizations of the random $\zeta_{n}$. Figure 2 shows the exponential localization for several degrees of rando:nness, $\Delta$ (this is figure 5.1 of Ref. 4).

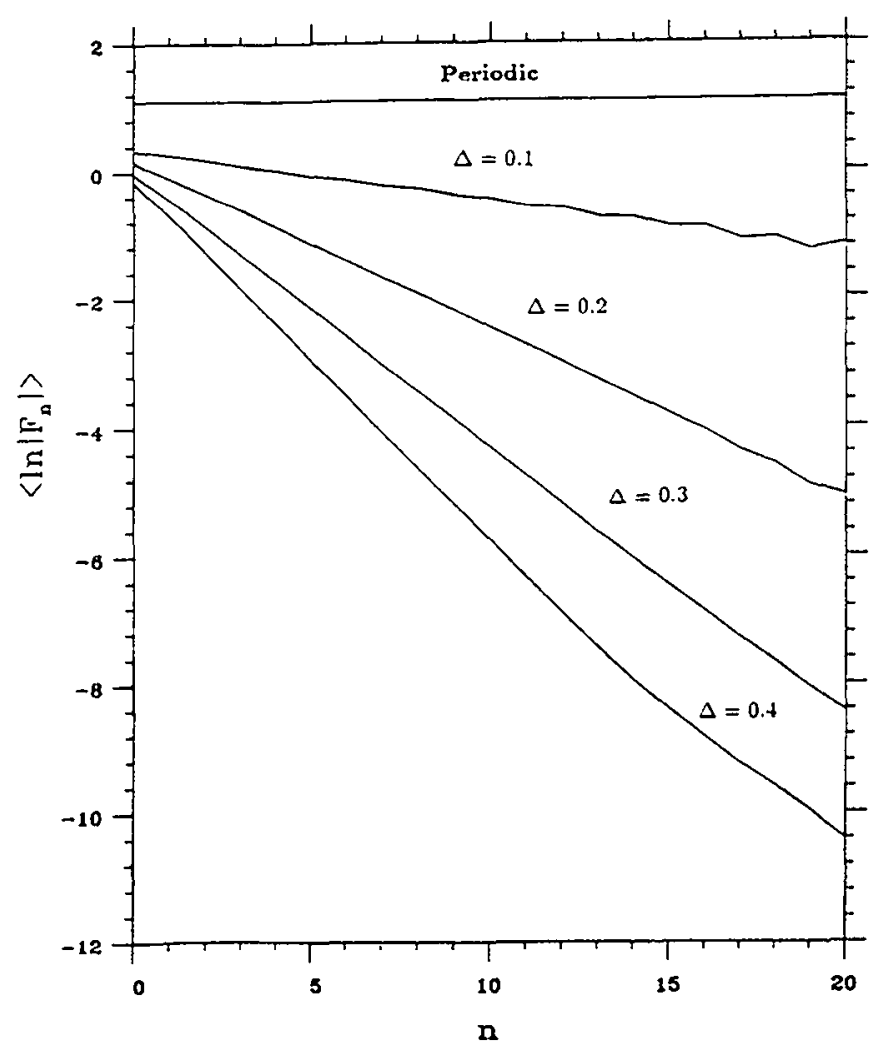

Figure 2. Anderson localization at a frequency which would lie at the middle of the first pass band of the periodic structure. Plane subs:onic wave coupling only. Values of $\Delta$ indicate different degsees of irregularity. (Fronı Ref. 4.) 
If, however, the long-range component is also included in the Green's function, then the exponential Anderson localization is short-circuited and replaced by algebraic decay - just as in the stop band. of the periodic array. The long-range algebraic decay is then almost the same (in absolute level at each bay) wha ever the value of randomness $\Delta$. We are currently working on a theory for this effect, and in particular for an understanding of the features of the long-range component of $G(x)$ that make the long-range decay of the $F_{n}$ so insensitive to structural irregularity.

\section{Acknowledgement}

All of the work described here has been consistently supported by the United States Office of Naval Research, through a program administered by Dr Phillip Abraham, Code $1132 \mathrm{SM}$; that support is gratefully acknowledged.

\section{References}

1. Crighton, D.G. \& Oswell, J. Phil. Trans. R. So:. Lond. A (1991), 335, 557-592.

2. Oswell, J. \& Crighton, D.G. submitted to Proc R. Soc. Lond., (1992).

3. Crighton, D.G. Proc. R. Soc. Lond. A. (1984), 394, 405-436.

4. Sobnack, M.B. Fluid loading and Anderson loc:alization. Ph.D. thesis, University of Cambridge (1991).

5. Spivack, M. Proc. R. Soc. Lond. A. (1991), 435, 615-634.

6. Crighton, D.G. J. Sound Vib. (1989), 133, 1-27.

7. Hodges, C.H. J. Sound Vib. (1982), 82, 411-421. 